\title{
Correlative Light, Electron, and Ion Microscopy for the Study of Urinary Tract Infection Pathogenesis
}

\author{
James A.J. Fitzpatrick ${ }^{1}$, Matthew S. Joens ${ }^{1}$, Valerie P. O’Brien ${ }^{2}$ and Scott J. Hultgren ${ }^{2}$ \\ 1. Washington University Center for Cellular Imaging, Washington University School of Medicine, Saint \\ Louis, USA \\ 2. Department of Molecular Microbiology and Center for Women's Infection Disease Research, \\ Washington University School of Medicine, Saint Louis, USA
}

Urinary tract infections (UTI) are common and costly infectious diseases, afflicting more than half of all women [1] and contributing to increasing medical costs and the spread of multi-drug resistant bacteria $[2,3]$. Bladder microscopy has been an essential tool for elucidating the pathogenesis of UTI caused by Escherichia coli [4-6]. Indeed, the discovery that E. coli forms intracellular bacterial communities (IBCs) within the bladder epithelium came from histology, fluorescence microscopy, scanning electron microscopy (SEM), and freeze fracture analysis of infected bladders [4, 7]. However, many aspects of host-pathogen interactions in UTI remain poorly understood, including: the behavior of E. coli within the IBC; the impact of host genetic background on susceptibility to infection; the causes and consequences of epithelial cell death and exfoliation in response to infection; the pathogenesis of lessstudied uropathogens, especially Gram-positive bacteria; and the changes to the bladder epithelium imparted by urinary catheterization. Although much research has been done in previous years, improved microscopy methods are needed in order to help answer these questions.

Notably, we have pioneered correlative light and electron microscopy on whole bladders infected with GFP-overexpressing E. coli to investigate IBC ultrastructure and assess the bacterial components necessary for functional IBCs. In order to correlate these structures across multiple microscopy modalities, three important conditions had to be met in order to minimize preparation-related artifacts and a to precisely correlate sample positions across multiple microscopes: 1) Since the targeting of IBCs required fluorescence microscopy, additional stains used for fiducial marking of features across both microscopes such as cell borders or nuclei needed to reach their targets without the use of permeabilizing agents or detergents, as these would disrupt the epithelial surface for SEM/ion beam analysis. 2) All fluorescence imaging had to be done with the samples still in their hydrated state to maintain fluorescence, while SEM imaging had to be performed with dehydrated samples under high vacuum conditions. 3) The samples needed to be mounted to a device with static features that could be visualized on both microscopes for Cartesian coordinate mapping and such that both the device and the tissue could withstand dehydration and avoid any forms of distortion or shrinkage. To meet all of these conditions, we created a single direction processing pipeline that started with samples being adhered to indium tin oxide coated coverslips that featured metal-deposited fiducial marks on three corners for position tracking [8]. These bladders were then sandwiched between a second coverslip and a spacer and analyzed on a laser scanning confocal microscope. The samples were then loaded into a critical point drier without any secondary staining or dehydration steps and subsequently coated with $6 \mathrm{~nm}$ of iridium. Following coating, the coverslips were then loaded into a focused-ion beam SEM (FIB-SEM). IBCs were located by using the fiducial points on the coverslips and the Zeiss Shuttle and Find algorithm to move the stage to the precise location of where the confocal images were taken. A single SEM image was then acquired and aligned to the confocal data using extracellular markers (Fig. 1). Once an IBC was confirmed by a bright GFP-positive region and lack of E. coli on the surface of the cells, we then utilized the gallium beam of the FIB to expose $(30 \mathrm{KeV}, 7 \mathrm{nA})$ and polish $(30 \mathrm{KeV}, 50 \mathrm{pA})$ the 
orthogonal face of the IBC-containing cell. This hole was then imaged at low voltage on the SEM to investigate the interior morphology (Fig. 2). Although this method is still in its infancy, further improvement in sample processing will improve speed of data acquisition and will assist in producing enough data to generate a true phenotype of IBCs for multiple clinical E. coli isolates. Using microscopy to increase our understanding of UTI pathogenesis and host response may ultimately lead to new drug targets and better outcomes for patients.

\section{References:}

[1] B. Foxman, Nature reviews. Urology 7 (2010), p. 653

[2] B. Foxman, Infectious disease clinics of North America 28 (2014), p. 1

[3] V. P. O'Brien, et al, Microbiology spectrum 4 (2016)

[4] M. A. Mulvey, et al, Science 282 (1998), p.1494

[5] G. G. Anderson, et al, Science 301 (2003), p. 105

[6] S. S. Justice, et al, Proc Natl Acad Sci 101 (2004), p. 1333

[7] M. A. Mulvey, J. D. Schilling and S. J. Hultgren, Infect Immun 69 (2001), p. 4572

[8] M. S. Lucas, Methods in Cell Biology 111 (2012), p. 325
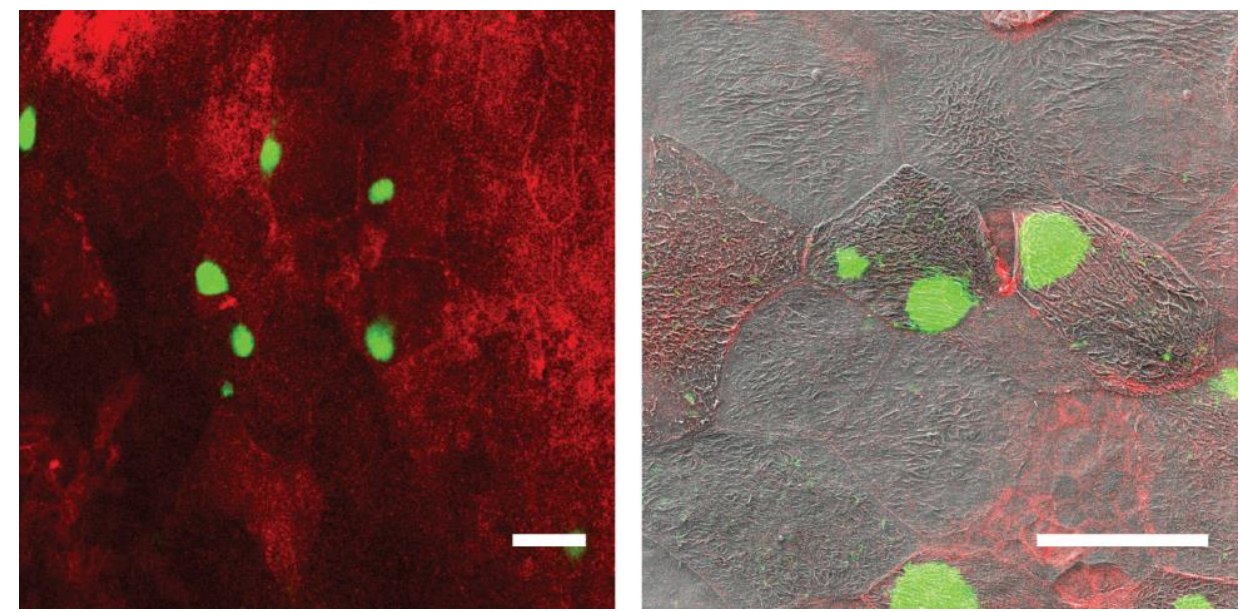

Figure 1. Confocal stack showing E. coli (green) colonization of bladder epithelium. After stage position correlation, the actin channel (red) was used to align the two datasets by matching cell borders. Scale: $100 \mu \mathrm{m}$
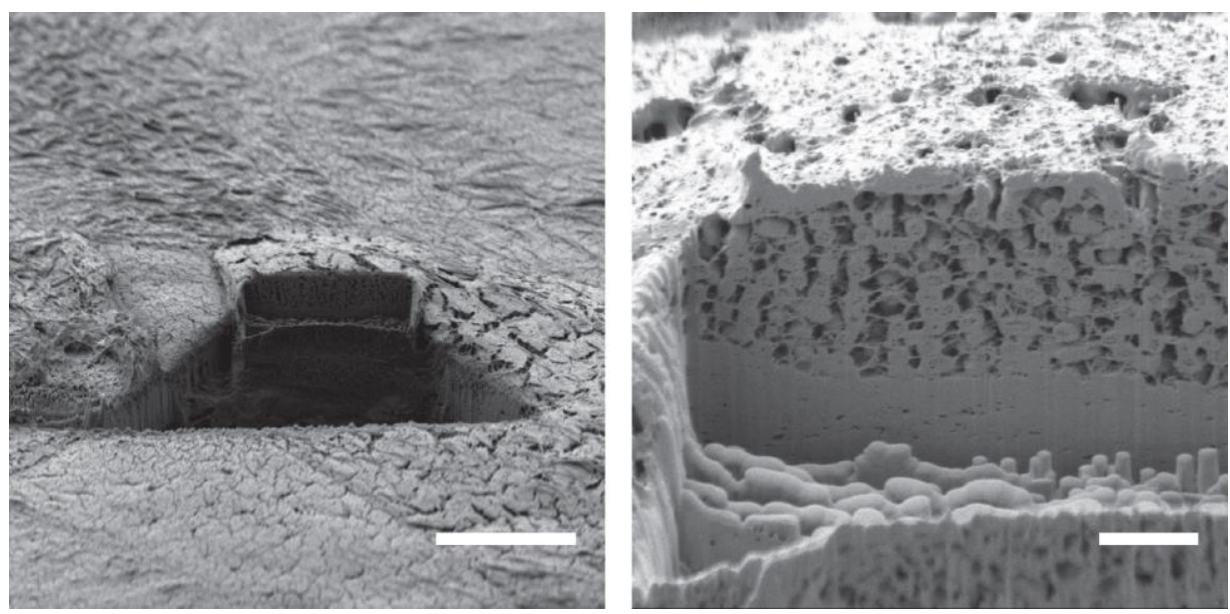

Figure 2. Once an IBC was identified, the cell interior was exposed using a gallium-FIB to investigate the interior morphology. Scale: $25 \mu \mathrm{m}$ (left), $2.5 \mu \mathrm{m}$ (right) 